\title{
Video Frames Edge Detection of Red Blood Cells: A Performance Evaluation
}

\author{
Karrar Neamah Hussein \\ Dir. Scholarship \& Cultural Relations \\ Baghdad, Iraq \\ karrarneamah@yahoo.com
}

Recived : 22\11\2017

Revised : 3\12\2017

Accepted : 612\2017

Available online : 27/1/2018

DOI: 10.29304/jqcm.2018.10.1.347

\begin{abstract}
The boundary structure (shape and size) and numbers of Red Blood Cells (RBCs) play a significant role in controlling human physiology. Several blood related diseases including sickle hemoglobin, polycythemia, thalassemia, anemia, and leukemia that occur due to the alteration of RBCs structures need precise detection, analysis, and subsequent inhibition. Diverse boundary analyses that have already been developed to detect the frames edge of blood samples remained ineffective. Thus, an automated system needs to be designed for exact detection of the RBCs edges. The edges being the main features of image often provide vital information to separate regions within an object or to detect changes in illumination. Accordingly, edge detection is regarded as an important step in the analysis of images and extraction of valuable information. In this paper, we evaluated the performance of video frames edge detectors and compared it with different gradient based techniques including Robert, Sobel, Prewitt and Canny, Zero-crossing (Laplacian, Gaussian and fuzzy logic) by applying them on video of RBCs. Two criteria such as mean square error (MSE) and peak signal to noise ratio (PSNR)were exploited to achieve good quality of video frames edge detectors for RBCs. Canny edge and fuzzy logic based techniques revealed the optimum performance towards the detection of video frames edge of RBCs.
\end{abstract}

Keywords: Edge detection, Fuzzy logic, Video frames edge, MSE, PSNR. 


\section{Introduction}

In recent times, digital image processing have been received a great attention in diversified fields [1]. Including computer vision office, industrial automation, face detection, features detection, lane departure warning system, remote sensing, natural resources survey, management, criminology, astronomy, meteorology and medical imaging [2]. Cumulative use of digital imaging systems has contributed significantly in the domain of medical diagnostics and greatly expanded in health care sector. Thus, computer aided diagnostic appeared as a routine means in clinical field [3]. Therefor rapid advancement in the electronic and photonic technology has allowed the invention of sophisticated diagnostic tools including Magnetic Resonance Imaging (MRI) or Computed Tomography (CT). Initially developed analogue imaging methods such as endoscopy or radiography are presently supported with digital sensors [4]. Despite such progress, a fully automated and accurate technique for RBCs edges detection is far from being developed.

Digital Images (DIs) are comprised of individual pixels, wherein the discrete color values or brightness are assigned. Using appropriate information communication networks and protocols, such DIs can be processed efficiently and made accessible at several places simultaneously. These protocols include picture archiving and communication systems (PACS) as well as the digital imaging and communications in medicine (DICOM) [5]. In medicine, the DI processing is now used for analyzing a significant volume of images to achieve high quality information for disease diagnosis and subsequent treatment. This helps considerably to the physicians, researchers, and medical practitioners to derive more diagnostically useful information [6].

Numerous blood related human diseases that occur from RBCs structural changes need to be detected, analyzed, and cured. RBCs are rich in iron-containing biomolecule called hemoglobin which bind oxygen and responsible for the red color of the blood. Approximately 2.4 million new erythrocytes are produced per second in an adult [7]. Human diseases are expressed through the abnormality or anomaly of RBCs. Changes in the RBCs shape, size, or number often cause diseases such as Sickle Hemoglobin, Sickle cell disease, Polycythemia, and Thalassemia. In this regard, an efficient blood samples frames edge detection tool is prerequisite. The image edges that render central information require careful evaluation.

In this paper, we assessed the performance of video frames edge detectors for RBCs and compared it with various existing art-of-the techniques. RBCs being the most significant components of human blood for delivering oxygen to the body tissues are considered in the present study.

\section{Edge Detection Techniques for Red Blood Cells [RBCs]}

Over the years, diverse RBCs edges detection techniques have been developed such as Robert, Sobel, Prewitt, Laplacian of Gaussian (LoG), Canny and Fuzzy Logic (FL). It is vital to focus on the various RBCs edges detection preprocessing steps to make a systematic comparison of the resulting edges. In the present method, first the videos of red blood cells are utilized under a microscope. Then, the input video files are converted into frames (still images). 
Afterward, the noise from these frames is removed using median filter [8]. Next, the thresholding method is used to change these images into grayscale with a resolution of $(3 \cdot 0 \times 2 r 0)$ pixels. Outcomes from each of the abovementioned edge detection technique are compared using two criteria. The quality and efficiency of these edge detector schemes are evaluated in terms of mean square error (MSE) and peak signal to noise ratio (PSNR). Figure 1 depicts the basic architecture of the edge detection scheme used in this study.

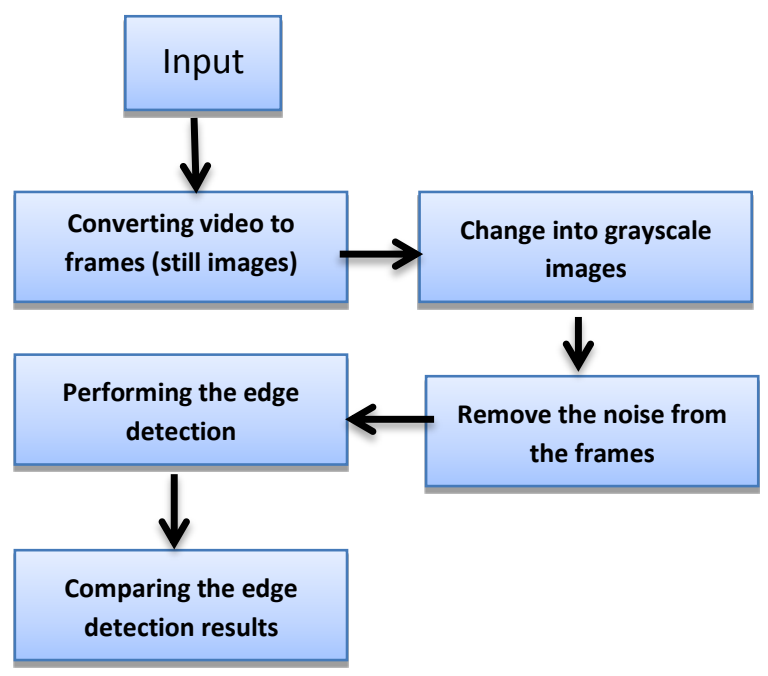

Figure 1: Schematic presentation showing the workflow for edge detection scheme using video file.

Median filter has been used to remove the noise, which is very useful for digital image processing applications. It consists of a window slides through the data and the median value inside the window acts as output of the filter. Being nonlinear in nature, median filter possesses several advantages including the maintenance of the edge and efficiency of noise removal with strength against impulsive type noise [9].
It is considered as one type of smoothing techniques similar to linear Gaussian filtering. Although all such filters are efficient in eliminating the noise in the smooth regions of the signal but they affect negatively at the edges. Thus, to preserve the properties of the video frames edges the median filter is selected [10].

\section{Development of Edge Detectors}

Edge detection process can reduce the amount of data in an image by simultaneously preserving the structural information involving object boundaries. Due to these reasons edge detectors are greatly beneficial in image analysis operations and object recognition [11]. Presently, several algorithms are available for edges detection, wherein each one has some advantages and disadvantages over the others. For instance, Robert, Sobel and Prewitt operators are based on the intensity gradient magnitude calculation at each image pixel. In such schemes, the gradient value is compared to the threshold value. If the value of the gradient is higher than a threshold value then a pixel location is classified as an edge, otherwise the pixel location is specified as not an edge [12].

The Laplacian of Gaussian operators are applied by choosing one mask and making a convolution to the original image with the mask. The positive or negative sign of the outcome from two adjacent pixel locations renders directional information in terms of brighter side of the edge. Subsequently, zero crossing is utilized to determine the alteration through zero in the values of the edges points [13]. Wide usage of these operators is limited by their sensitiveness to noise. To surmount the noise problems, Canny proposed a method where image convolution was used with the first order derivatives of Gaussian filter for smoothing in the local gradient direction followed by edge detection and thresholding[14]. 


\subsection{Roberts Edge Detector}

This is a simple and fast detection scheme for 2-D spatial gradient measurement on the image. It selects the areas of high spatial gradient, which often conform to the edges. The input of the Roberts operator is a grayscale image, whereas each pixel value in the output specifies the estimated absolute quantity of spatial gradient of the original input image at that point $[11,12]$. Theoretically, Roberts's operator is comprised of a pair of $(2 \times 2)$ convolution masks as illustrated in Figure 2.

\begin{tabular}{|l|l|}
\hline $1+$ & 0 \\
\hline 0 & -1 \\
\hline
\end{tabular}

Gy

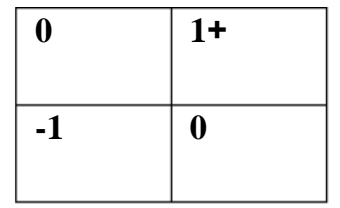

$G x$
Figure 2: Roberts Cross convolution masks.

These masks have been proposed to respond the maximum of edges working in $45^{\circ}$ to the pixel grid, where one mask is used for each of the two vertical orientations. The masks can be used individually to the input image for separate measurements of the gradient contents in each direction (called $G x$ and $G y$ ) [12]. Mathematically, Roberts's operator is defined as:

$G(x, y)=\left\{[\sqrt{f(x, y)}-\sqrt{f(x+1, y+1)}]^{2}-[\sqrt{f(x+1, y)}-\sqrt{f(x, y+1)}]^{2}\right\}^{\frac{1}{2}}$

\subsection{Sobel Edge Detector}

This operator performs a 2-D spatial gradient measurement on an image. It focuses on the areas of high spatial gradient that match to the edges. Generally, it is used to estimate the absolute gradient magnitude at each point in an original grayscale image. This operator is consisted of a pair of $(3 \times 3)$ convolution masks as displayed in Figure 3, where the first mask is just the second one rotated by $90^{\circ}$.Sobel operator resembles the Roberts cross operator.

$$
G x=\left[\begin{array}{lll}
-1 & 0 & +1 \\
-2 & 0 & +2 \\
-1 & 0 & +1
\end{array}\right] \quad G y=\left[\begin{array}{ccc}
+1 & +2 & +1 \\
0 & 0 & 0 \\
-1 & -2 & -1
\end{array}\right]
$$

Figure 3: Sobel convolution masks.

These kernels have been proposed to respond the maximum of edges working horizontally and vertically relative to the pixel grid with one mask for each of the two vertical directions. These masks can be used individually to the input image, to obtain discrete measurements of the gradient component in each direction (called $G x$ and $G y$ ). It is used to calculate the absolute magnitude of the gradient at each pixel and the direction of that gradient [14]. The edge magnitude and the edge direction are defined via the relation:

$$
\begin{aligned}
& \text { EDGE MAGNITUDE }=\sqrt{S_{1}{ }^{2}+S_{2}{ }^{2}} \\
& \text { EDGE DIRECTION }=\tan ^{-1}\left(\frac{s_{1}}{s_{2}}\right)
\end{aligned}
$$

\subsection{Prewitt Edge Detector}

Prewitt operator is used for detecting two types of edges such as Horizontal and Vertical. This operator produces an image, wherein the higher grayscale value refers to the existence of an edge between two objects. This operator is similar to the Sobel operator but with different mask coefficients. Besides, it contains a pair of $(3 \times 3)$ convolution masks as follows:

$G x=\left[\begin{array}{lll}-1 & 0 & +1 \\ -1 & 0 & +1 \\ -1 & 0 & +1\end{array}\right] \quad G y=\left[\begin{array}{ccc}+1 & +1 & +1 \\ 0 & 0 & 0 \\ -1 & -1 & -1\end{array}\right]$

Figure 4: Prewitt convolution masks. 
At each pixel location, two numbers are found with p1 (corresponding to the result from the vertical edge mask) and p2 (corresponding to the result from the horizontal edge mask). These numbers are used to determine the edge magnitude and the edge direction using the expression[15].

$$
\begin{aligned}
& \text { EDGE MAGNITUDE }=\sqrt{P_{1}{ }^{2}+P_{2}{ }^{2}} \\
& \text { EDGE DIRECTION }=\tan ^{-1}\left[\frac{P_{1}}{P_{2}}\right]
\end{aligned}
$$

\subsection{Laplacian of Gaussian}

The Laplacian of an image focuses on the regi ons of intensity that has fast change and thus useful for detecting the edges. To minimize the noise sensi tivity of an image the Laplacian is often utilized wit $\mathrm{h}$ a Gaussian Smoothing filter. Usually, this operato $r$ uses a single gray level image as input and produc es a gray level image as output. The Gaussian functi on is used as low-pass smoothing filter of the image and the Laplacian operator acts as high-pass filter a ccording to the second derivative of zero to detect $t$ he edges. Gaussian filter function is defined as [16]:

$$
\operatorname{LOG}(x, y)=-\frac{1}{\pi \sigma^{4}}\left[1-\frac{x^{2}+y^{2}}{2 \sigma^{4}}\right] e^{-\left(\frac{x^{2}+y^{2}}{2 \sigma^{4}}\right)}
$$

After the noise minimization from the input image using Gaussian filter, the Laplacian convolution masks are applied on the image. Afterward, the threshold value is calculated. Finally, the zero crossing method is applied to determine the change through zero in the values of the edges points [15]. Generally, three small masks are used as depicted in Figure5.

$$
\left[\begin{array}{ccc}
0 & -1 & 0 \\
-1 & 4 & -1 \\
0 & -1 & 0
\end{array}\right] \quad\left[\begin{array}{ccc}
-1 & -1 & -1 \\
-1 & 8 & -1 \\
-1 & -1 & -1
\end{array}\right] \quad\left[\begin{array}{ccc}
-2 & 1 & -2 \\
1 & 4 & 1 \\
-2 & 1 & -2
\end{array}\right]
$$

Figure 5: Laplacian convolution masks.

\subsection{Canny Edge Detector}

This edges detection technique is based on three criteria. After converting an input image into gray image, the Gaussian convolution is used for noise reduction and to smooth image. The maximum value of the first derivative corresponds to the minimum of the first derivative. It means that the points with radical change of gray-scale (strong edge) and points with simple change of grayscale (weak edges) correspond to the second derivative zero-crossing point. Thus, these two thresholds are used to detect strong edges and weak edges. Actually, the Canny algorithm is not exposed to noise interference due to its true weak edges detection capacity $[14,17]$.

$G^{\prime \prime}(X)=\left(-\frac{X}{\sigma^{2}}\right) e^{-\left(\frac{X^{2}}{2 \sigma^{2}}\right)}$

\subsection{Fuzzy Logic}

Fuzzy logic (FL) is defined as a superset of classical (Boolean) logic which are expanded to handle the concept of partial time values between completely true and completely false. It deals with reason that is approximate rather than exact, where variables of FL may have a true value that ranges between 0 and 1. Certainly, FL is a powerful problem solving technique with diverse applicability, especially in the fields of control and decision making [18].

Fuzzy image processing can be defined as a collection of all approaches that recognize and represent the images. The representation and processing of image based on the chosen fuzzy method and on the problem under consideration. In most of these techniques, adjacent points of pixels are assumed in some classes and then fuzzy system inference are implemented using suitable membership function defined for each class. 
Fuzzy image processing is comprised of three main stages such as image fuzzification, fuzzy technique, and image defuzzification. The fundamental step of fuzzy image processing is the modification of membership values. Therefore, the coding of image data (fuzzification) and decoding of the results (defuzzification) are the vital steps for image processing using FL [19].

Edges in the images that constitute a significant gray level change needs precise detection. For edge detection a set of nine pixels which is a part of $(3 \times 3)$ or $(5 \times 5)$ window of an image to a set of fuzzy conditions are useful to focus on all the edges of an image. In the fuzzy edge detection, points affiliated to a common area must be white, otherwise they must be black, where black and white are the fuzzy variables. For integrated area concept, the intensity variation is considered between the pixel at the kernel of the neighborhood and its neighbors. This helps to compare the pixels values, which are present on the edge in a gray scale image. Figure 6 provides a graphical representation of the rules used in FL where $\mathrm{ZE}$ is the variety of the independent variables of the fuzzy set. The box leveled $Z_{5}$ represents the intensity of the center pixel which is determined to be the output value with WH (white) or BL (black) [20].

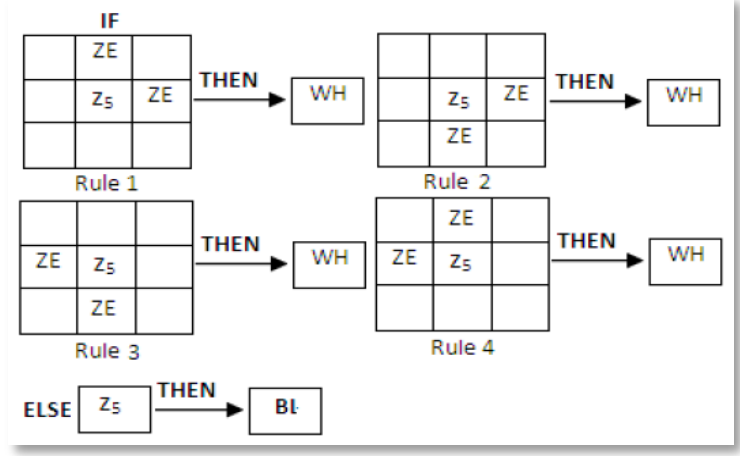

Figure 6:Schematic representation showing the rules of FL.

\section{Parameters for Performance} Evaluation of the Edge Detection Method

\subsection{Mean Squared Error (MSE)}

Generally, is defined as the difference between the estimated and the true value of the quantity under study. MSE can be match to the predicted value of the squared error loss. The MSE (estimated) of the predictor is defined as:

$M S E=\frac{1}{N} \sum_{i=0}^{n}\left(\hat{Y}_{i}-Y_{i}\right)^{2}$

where $\hat{Y}$ is a vector of $n$ predictions, $Y$ is the vector of the true values, and $N$ is the total number of realiz ations. The MSE of the estimator is defined as:

$\operatorname{MSE}(\breve{\boldsymbol{\theta}})=E\left[(\check{\theta}-\theta)^{2}\right]$

where $\theta$ is the computed magnitude of the parameter for a given sample (sample-dependent)and $\check{\theta}$ is the corresponding unknown value. This definition is based on the unidentified parameter. The MSE in this context is a property of an estimator (of a way to get an estimate) the MSE is equivalent to the total of the difference and the squared bias of the estimator or of the expectations, in the condition of the MSE of an estimator [21].It yields: $\operatorname{MSE}(\check{\theta})=\operatorname{Var}(\check{\theta})+(\operatorname{Bias}(\check{\boldsymbol{\theta}}, \theta))^{2}$

\subsection{Peak Signal-to-Noise Ratio (PSNR)}

Commonly, the PSNR is used to measure the quality of reconstruction of loss compression codecs where the signal is the main data and the noise is the error that is introduced by compression. Therefore, when comparing compression codecs, PSNR approximates human perception of reconstruction quality. Though a higher PSNR typically refers to restructuring of higher quality but in some conditions it differs [22]. 
The MSE and the PSNR are the two metrics used to measure error standards for determining the image quality or comparing among images. The MSE determines the cumulative squared error between the original image and the resulting image. The PSNR is a measure of the peak error. A lower MSE value indicates an accurate image features. To obtain the PSNR values, first the MSE values of the block are computed using:

$$
M S E=\frac{\sum_{M, N}\left[I_{1}(m, n)-I_{2}(m, n)\right]^{2}}{M * N}
$$

Where $M$ and $N$ represent the number of rows and columns in the input images, respectively. The block calculates the PSNR using the relation:

$$
P S N R=10 \log 10\left(\frac{R^{2}}{M S E}\right)
$$

Where $\mathrm{R}$ is the maximum fluctuation in the input image data type [23]. These popular standards are chosen and the performance evaluation of RBCs video frames edges detection schemes.

\section{Results and Discussion}

Experiments are conducted using MATLAB code to determine the quality and clarity of edge detection in the video image for RBCs boundaries. Two videos for RBCs under a microscope are considered. The blood samples are collected from laboratory. One sample contained normal RBCs with521 frames in 16 minutes and the other enclosed abnormal RBCs with 219 frames in 9 minutes. Each video consisted of many frames which are randomly chosen and applied in the experiments. First, the videos are changed to frames and then the colored frames are changed to grayscale images. Next, the noise from the frames is removed using median filter to preserve the edges.
Afterward, all the aforementioned edge detectors are applied on the frames. The edge pixels to size ratio of the image, MSE and PSNR for each operator is calculated to determine the effectiveness of the operator in detecting the edges of the RBCs. Experimental results are summarized in Table 1.Figure 7 compares the original color image with the converted gray scale image after using the median filter. Figures 8-13 compares the outcome of various edge detectors with MSE for normal RBCs frame number 250.

Table 1: Comparative analysis among various edge detectors for the second sample (frame number 250) of normal RBCs under microscope.

\begin{tabular}{|l|c|c|c|}
\hline \multicolumn{1}{|c|}{ Edge } & $\begin{array}{c}\text { Ratio of edge pixels } \\
\text { to size of image (\%) }\end{array}$ & MSE & PSNR \\
\hline Roberts & 29.22 & 0.0321 & 63.2675 \\
\hline Sobel & 31.74 & 0.0282 & 63.6363 \\
\hline Prewitt & 31.74 & 0.0270 & 63.7998 \\
\hline LoG & 48.40 & 0.0268 & 63.8607 \\
\hline Canny & 75.43 & 0.0265 & 63.8381 \\
\hline Fuzzy & 64.14 & 0.0226 & 63.5697 \\
\hline
\end{tabular}

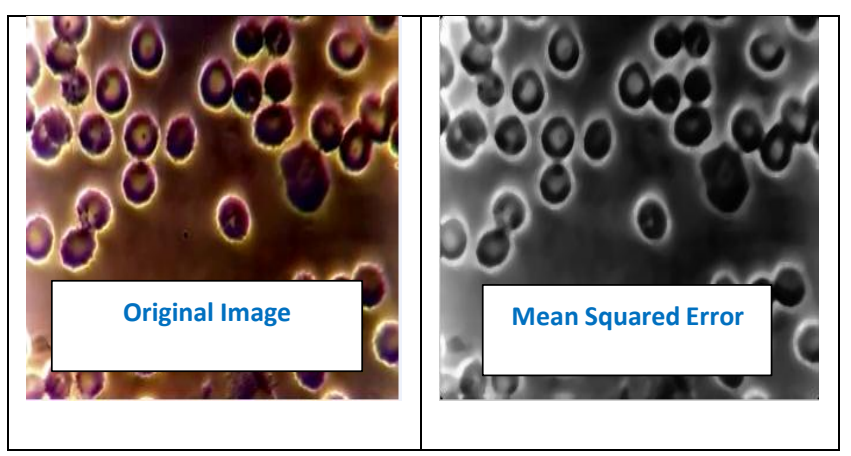

Figure 7: The original image and median filter image for normal RBCs frame number 250. 


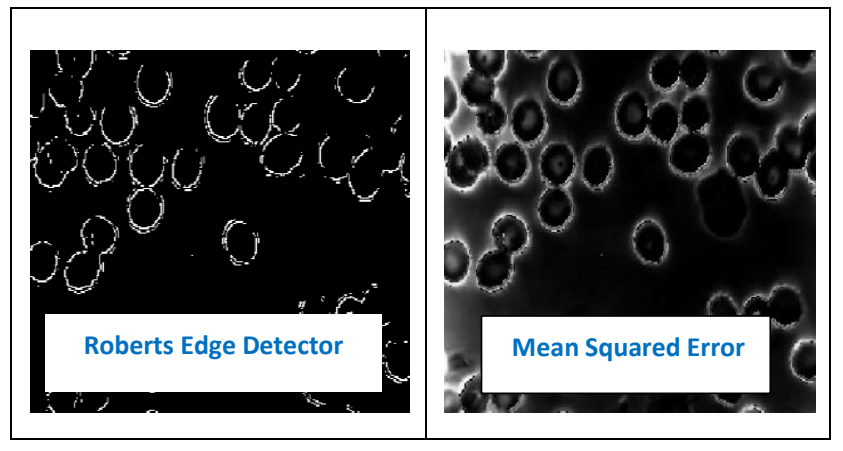

Figure 8: Roberts edge detector and MSE for normal RBCs frame number 250 .

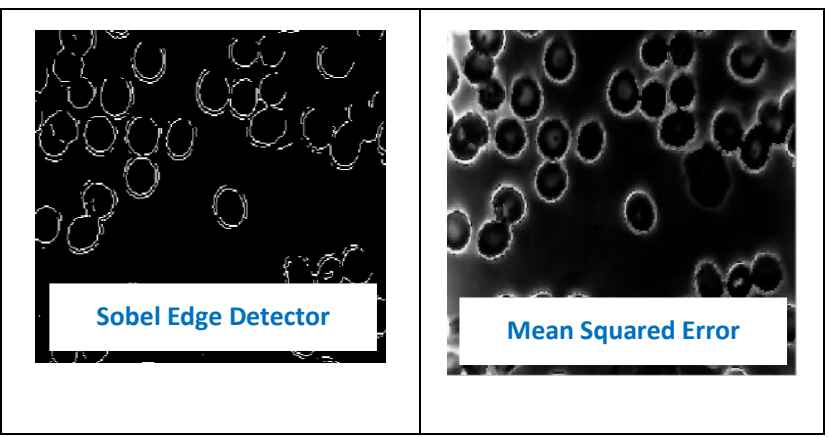

Figure 9: Sobel Edge Detector and MSE for normal RBCs frame number 250 .

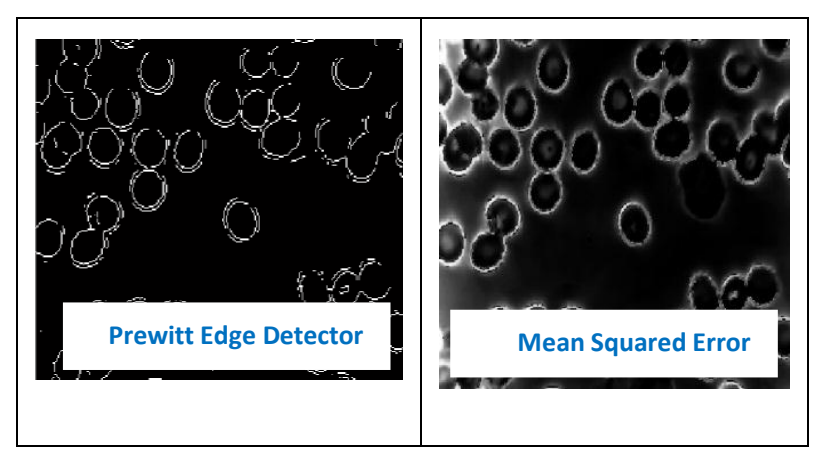

Figure 10: Prewitt Edge Detector and MSE for normal RBCs frame number 250.

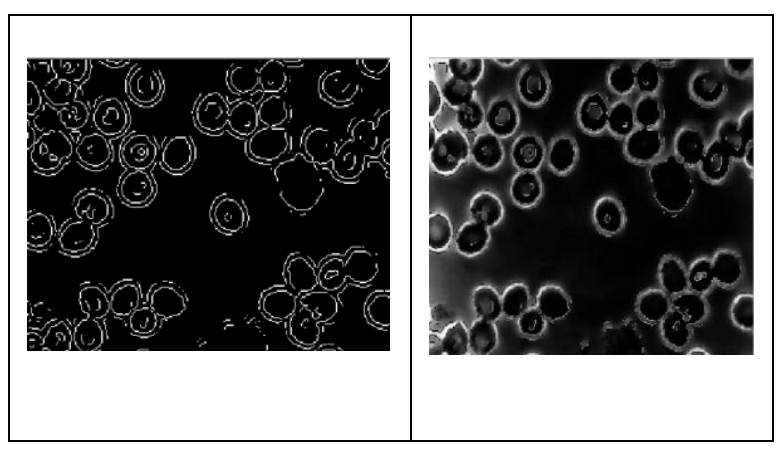

Figure 11:LoG Edge Detector and MSE for normal RBCs frame number 250.

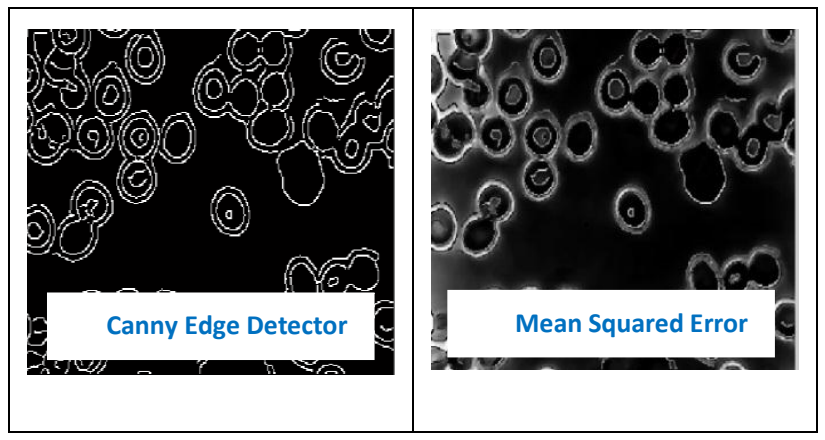

Figure 12: Canny Edge Detector and MSE for normal RBCs frame number 250 .

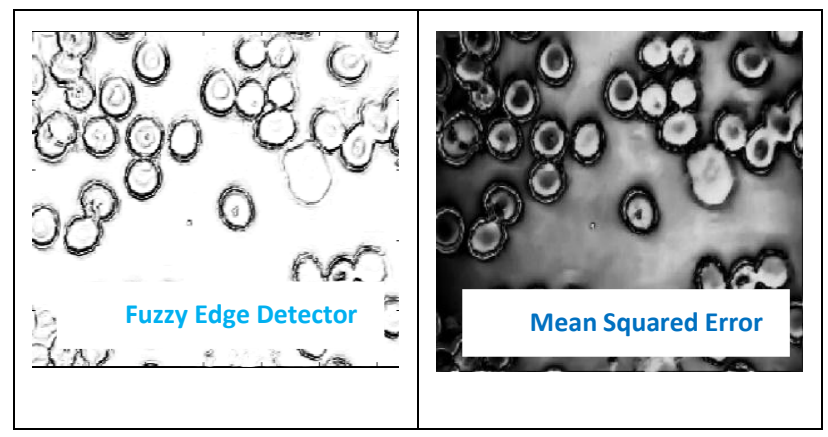

Figure 13: Fuzzy Edge Detector and MSE for normal RBCs frame number 250. 


\section{Performance Evaluation}

In the present study, two medical videos of Red blood cells (RBCs) are selected. The first one is for normal RBCs and the other is for abnormal RBCs. After converting these videos to frames, grayscale images are obtained and the noise is removed. Finally, all edge detectors are applied to these images. The original medical images (frames) of normal RBCs from video and subsequent removal of noise from the images using median filter. Figure 8 revealed a clear difference in terms of clarity. Similar observations are made for abnormal RBCs from video and for noise removed images using median filter. The images for edges which are obtained by applying Roberts, Sobel, Prewitt, LoG, Canny and fuzzy edges detectors on all RBCs frames and the calculated mean squared error displayed a clear difference among various method (Figures 8-13).

The results (Table 1) for the ratio of edge pixels to size of image, mean squared error (MSE) and peak signal-to-noise ratio (PSNR) for the resultant image revealed the improvement of one method over the other. An increase in the ratio of edge pixels to size of image indicated the enhanced efficiency of the edge detector and vice versa. Moreover, any lacking of MSE signified the decreased efficiency of the edge detector and vice versa. An increase in the PSNR signified the enhanced efficiency of the edge detector and vice versa. In the Roberts detector, the edges of the object are not smooth enough. Sobel detector produced less information of edges. Prewitt detector provided better edges than Sobel and Roberts.
Laplacian of Gaussian detector rendered useful information of edges, while Canny created better edges with higher PSNR values, which confirmed the superiority of the method in RBCs frames edge detection. The FL revealed great accuracy in detecting the edges of RBCs and achieved lowest values of MSE, indicating its lacking of error estimation in the edge detection. Furthermore, FL displayed some distinct features than others operators, without same limitations like fixed edge thickness and threshold. It is established that FL is flexible to implement where the edge thickness can simply be changed by changing the rules and output parameters.

\section{Conclusions}

This paper compared the performance of various schemes useful for RBCs video frames edge detection. Using edge detection, the sharp discontinuities are located, where such discontinuities are sudden changes in pixel intensity that distinguish the boundaries of objects in an image. The boundaries of RBCs are examined to determine whether they are normal or abnormal because it is known that several human diseases occur due to the change in the shape, size or number of RBCs. The efficiency of various operators in detecting the borders of RBCs is compared. MATLAB R2014a coding is used for frames of video and the computational results are analyzed in terms of MSE and PSNR. Based on the experimental results, the following conclusions are drawn:

i) Robert operator produced smooth edges of RBCs as compared to other operators. 
ii) Sobel provided less information of edges for RBCs than other operators. It is more sensitive to the diagonal edge is than the horizontal one. Although Sobel edges spurious but they are relatively less intense compared to original one.

iii) Roberts operator has missed a few edges and the Sobel operator detected thicker edges than Robert. This indicated that Sobel is slightly better than Roberts scheme.

iv) Prewitt operator rendered better information than Robert but is more sensitive to horizontal and vertical edges. All images of RBCs showed a difference in the ratios of pixels edges to Sobel and Prewitt. Prewitt and Sobel detectors approximately revealed same level of performance without significant difference.

v) Canny detector exhibited greatly smooth edges of RBCs and performs better than all others. Images evaluation showed that a low threshold produces the false edges and high threshold misses the important edges. Moreover, Canny for edge detection is computationally more expensive than Laplacian of Gaussian (LOG), Sobel, Prewitt, and Robert's operator because of the chosen three criteria. In short, vi) Canny revealed a better edge detection than other operators. The highest value of PSNR clearly showed that the edge detected image in Canny has enclosed less noise than other operators.

vii) Laplacian of Gaussian (LoG) provided quite similar information to Canny, but the edges are unsmooth.

viii) FL operator revealed lowest MSE, indicating its highest accuracy for video frames edges detection of RBCs. It is asserted that the Canny and FL detectors are appropriate to use as edge detectors for RBCs.

\section{References}

[1] Adrian, R. J. (2005). Twenty Years of Particle Image Velocimetry. Experiments in Fluids, 39(2), 159-169.

[2] National Research Council. (2013). Future us Workforce For Geospatial Intelligence. National Academies Press.

[3] Szabo, T. L. (2004). Diagnostic Ultrasound Imaging: Inside Out. Academic Press.

[4] Dougherty, G. (2009). Digital Image Processing For Medical Applications. Cambridge University Press.

[5] Deserno, T. M. (2010). Fundamentals of Biomedical Image Processing. In Biomedical Image Processing (Pp. 151). Springer Berlin Heidelberg.

[6] Mair, T. S., \& Kinns, J. E. N. (2005). Deep Digital Flexor Tendonitis in the Equine Foot Diagnosed By Low- Field Magnetic Resonance Imaging In The Standing Patient: 18 Cases. Veterinary Radiology \& Ultrasound, 46(6), 458466. 
[7] Underwood, E. (2012). Trace Elements in Human and Animal Nutrition. Elsevier. 2, 459-461.

[8] Cheng, E. D., Challa, S., \& Chakravorty, R. (2011). Parallel Microscopic Cell Image Segmentation and Multiple Fusions. International Journal of Signal And Imaging Systems Engineering, 4(2), 96-114.

[9] Jayaraj, V., \& Ebenezer, D. (2010). A New Switching-Based Median Filtering Scheme and Algorithm For Removal Of High-Density Salt And Pepper Noise In Images. Eurasip Journal on Advances in Signal Processing, 2010(1), 690218.

[10] Arias-Castro, E., \& Donoho, D. L. (2009). Does Median Filtering Truly Preserve Edges Better Than Linear Filtering. The Annals of Statistics, 11721206.

[11] Kaur, E. K., Mutenja, V., \& Gill, E. I. S. (2010). Fuzzy Logic Based Image Edge Detection Algorithm in Matlab. International Journal of Computer Applications, 1(22), 55-58.

[12] Nadernejad, E., Sharifzadeh, S., \& Hassanpour, H. (2008). Edge Detection Techniques: Evaluations and Comparison. Applied Mathematical Sciences, 2(31), 1507-1520.

[13] Huang, C. Y., \& Wu, M. J. (2006). Image Segmentation. Ece, 533, 1-3.

[14] Vincent, O. R., \& Folorunso, O. (2009, June). A Descriptive Algorithm For Sobel Image Edge Detection. In Proceedings of Informing Science \& It Education Conference (Vol. 40, Pp. 97-107).

[15] Bhatia, M., Yadav, D., Gupta, P., Kaur, G., Singh, J., Gandhi, M., \& Singh, A. (2013, September). Implementing Edge Detection For Medical Diagnosis of A Bone In Matlab. In Computational Intelligence and Communication Networks, 2013 5th International Conference on (Pp. 270-274). IEEE
[16] Vincent, O. R., \& Folorunso, O. (2009, June). A descriptive algorithm for sobel image edge detection. In Proceedings of Informing Science \& IT Education Conference (Vol. 40, pp. 97-107).

[17] Maini, R., \& Aggarwal, H. (2009). Study and Comparison of Various Image Edge Detection Techniques. International Journal of Image Processing , 3(1), 1-11.

[18] Mohanty, P. K., \& Parhi, D. R. (2013). Controlling the Motion of An Autonomous Mobile Robot Using Various Techniques: A Review. Journal of Advance Mechanical Engineering, 1(1), 24-39.

[19] Aborisade, D. O. (2011). Novel Fuzzy Logic Based Edge Detection Technique. International Journal of Advanced Science and Technology, 29(1), 75-82.

[20] Khaire, P. A., \& Thakur, N. V. (2012). A Fuzzy Set Approach For Edge Detection. International Journal of Image Processing, 6(6), 403-412.

[21] Hoerl, A. E., \& Kennard, R. W. (2000). Ridge regression: biased estimation for nonorthogonal problems. Technometrics, 42(1), 80-86.

[22] Gupta, M., \& Garg, A. K. (2012). Analysis of image compression algorithm using DCT. International Journal of Engineering Research and Applications (IJERA), 2(1), 515-521.

[23] Huynh-Thu, Q., \& Ghanbari, M. (2008). Scope of validity of PSNR in image/video quality assessment. Electronics letters, 44(13), 800-801. 


\title{
إطارات الفيديو كشف حافة خلايا الدم الحمراء: تقييم الأداء
}

\author{
كرار نعمه حسين \\ دائرة البعثات والعلاقات الثقافية \\ karrarneamah@tahoo.com
}

: (لمستخلص : (ل)

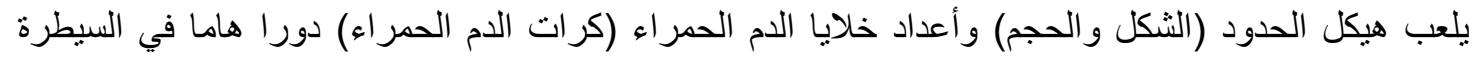

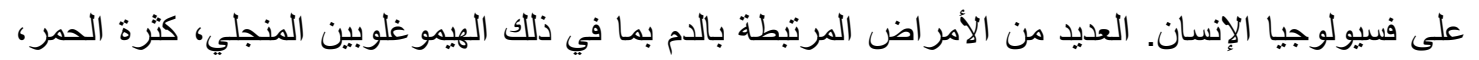

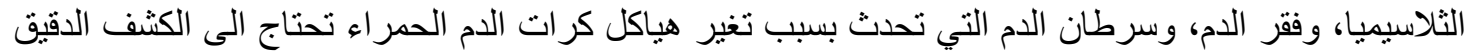

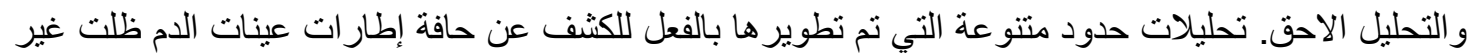

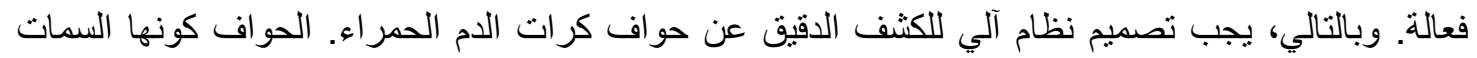

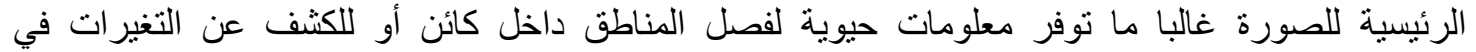

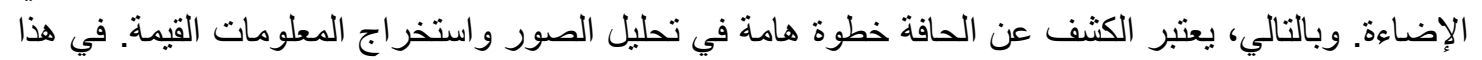

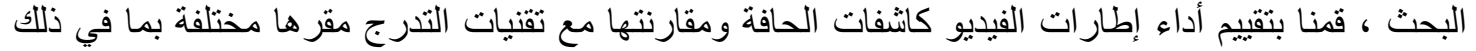

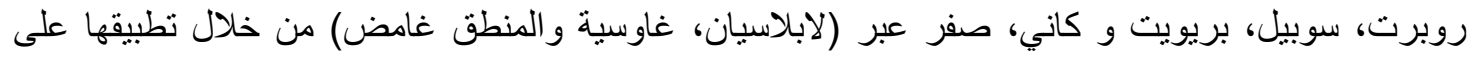

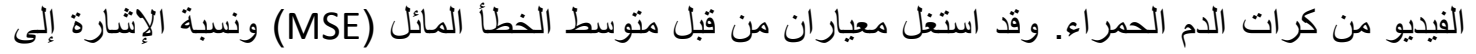

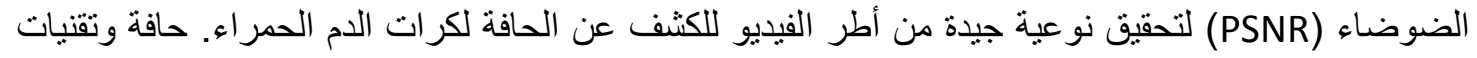

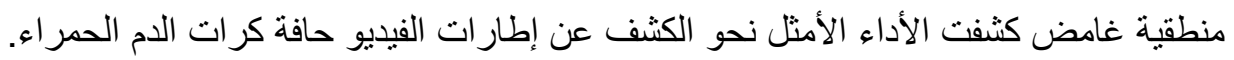

